\title{
Experiments on the mechanism of inducing transition between regular and Mach reflection
}

\author{
Christopher A. Mouton ${ }^{a)}$ and Hans G. Hornung ${ }^{\text {b) }}$ \\ Graduate Aeronautical Laboratories, California Institute of Technology, 1200 East California Boulevard, \\ MC 105-50, Pasadena, California 91125, USA
}

(Received 15 July 2008; accepted 7 November 2008; published online 18 December 2008)

\begin{abstract}
A study of the mechanism by which disturbances can cause tripping between steady-flow regular and Mach reflection in the dual-solution domain is presented. Computational results indicate that the disturbance shock created as a result of the impact of dense particles on one of the shock-generating wedges can cause transition from regular to Mach reflection. The disturbance shock may also be generated by direct energy deposition on the wedge. Estimates of the lower bound of the required energy for transition to occur are presented and compared to values obtained computationally. Experiments were performed at Mach 4.0 in a Ludwieg tube that has a test duration of $100 \mathrm{~ms}$. Proper starting of the flow necessitated operation with an upstream diaphragm and modifications in the dump tank. The reflection state was changed by rapid rotation of one of the shock-generating wedges. The flow in the facility is sufficiently quiet to permit entering the dual-solution domain to approximately its midpoint before spontaneous transition to the Mach reflection occurs. The short test time prompted a study of the effect of wedge rotation speed on the transition from regular to Mach reflection. Transition due to deposition of energy on one of the wedges was also examined by using a pulsed laser focused on one of the two wedges. Measurements of the minimum energy to bring about transition and of the rapid growth of the Mach stem to its steady-state are compared to numerical and theoretical predictions. (C) 2008 American Institute of Physics.
\end{abstract}

[DOI: $10.1063 / 1.3042261]$

\section{INTRODUCTION}

If a wedge is placed into a steady supersonic flow in such a way that its oblique attached shock impinges on a flat wall or symmetry plane parallel to the free stream, the shock turns the flow toward the wall and a reflected shock is required to turn the flow back to a direction parallel to the wall. When the shock angle exceeds a certain value, the deflection achievable by a single reflected shock is insufficient to turn the flow back to a direction parallel to the wall and transition to the Mach reflection is observed, in which a near-normal shock forms a triple shock point with the incident and reflected shock. An overview of shock reflection is given, e.g., by Ben-Dor. ${ }^{1}$

For a range of Mach number there exists a region where both regular reflections, see, for example, Fig. 11(a), with a single reflected shock, and the Mach reflection, see, for example, Fig. 11(e), are possible. This region is referred to as the dual-solution domain. Figure 1 shows some of the shock reflection domains in the Mach number-shock angle plane. The incident shock angle is denoted by $\alpha$ and the free-stream Mach number is denoted by $M$. The upper curve represents the condition where the flow behind the incident shock is sonic and any increase in incident shock angle would produce subsonic flow. The curve second from the top is known as the detachment condition. At incident shock angles above this curve regular reflection is not possible. The detachment

\footnotetext{
a)Electronic mail: mouton@alumni.caltech.edu.

${ }^{b)}$ Electronic mail: hans@galcit.caltech.edu.
}

condition can be expressed as a fifth-order polynomial in $\sin ^{2} \alpha$ and the coefficients are functions of $M$ and the ratio of specific heats $\gamma$. The exact form of the equation and coefficient values are given by Mouton. ${ }^{2}$ The curve second from the bottom is known as the von Neumann condition and is defined such that for incident shock angles less than this value direct Mach reflection is not possible. Finally, the lower curve is the Mach wave condition below which no shock exists.

The region of importance to the current work is the dualsolution domain, which is the area between the von Neumann condition and the detachment condition. The possibility for hysteresis in this dual-solution domain was first proposed by Hornung et $a l .^{3}$ It was suggested that if the dual-solution domain entered from below the von Neumann condition then regular reflection persists, and if it entered from above the detachment condition then the Mach reflection persists. In experiments performed at four values of $M$, Hornung and Robinson failed to observe hysteresis, and transition occurred at the von Neuman condition. Then, Ivanov et al. ${ }^{4}$ were able to demonstrate hysteresis in numerical computations, and later, in an extensive experimental campaign in several Russian wind tunnels, showed that it also occurs experimentally if the facility is sufficiently quiet. ${ }^{5}$ Others, notably Sudani et al., ${ }^{6}$ later demonstrated hysteresis in experiments. Theoretical arguments and the body of experimental work indicate that the stable reflection state in the dual-solution domain is the Mach reflection, and that the lower the level of disturbances is in the facility, the closer the 


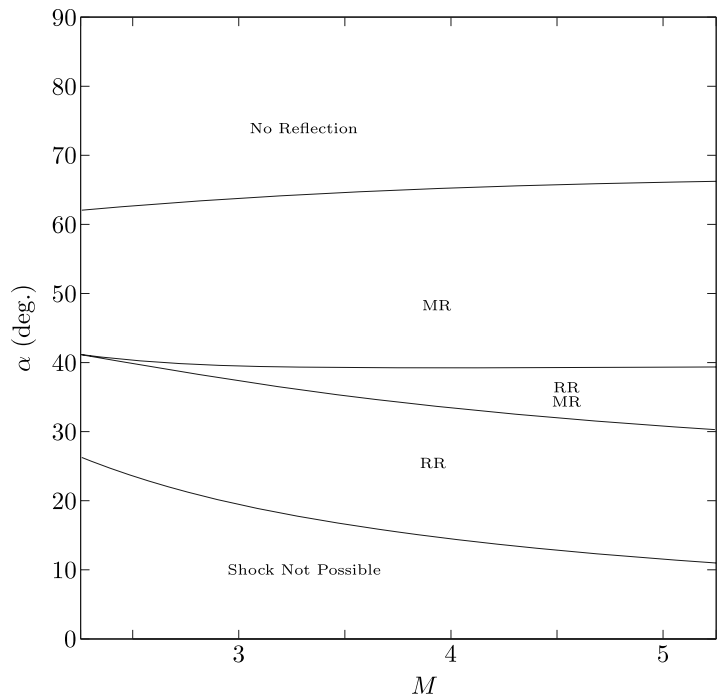

FIG. 1. Simplified shock reflection domain for $\gamma=1.4$, several more complex shock reflections have been omitted from the plot for illustrative purposes. RR and MR stand for regular and Mach reflections, respectively, and $\alpha$ is the incident shock angle.

detachment condition can be approached from below while retaining regular reflection.

In order to learn more about the mechanism by which disturbances can cause transition, it is necessary to know details about the disturbance. It is difficult and time consuming to characterize the nature and magnitude of the various disturbance types in a facility. The aim of the experiments described here is therefore to study the effect of deliberately introduced and well-defined disturbances in a flow in which the reflection state is initially regular reflection in the dualsolution domain.

\section{EXPERIMENTAL SETUP}

The experiments were conducted using the Ludwieg tube at the Graduate Aeronautical Laboratories of the California Institute of Technology (GALCIT). The details of the facility and the specific test setup are discussed in this section.

\section{A. Ludwieg tube}

A Mach 4 nozzle was designed, constructed, and taken into operation for the existing Ludwieg tube at GALCIT. The Ludwieg tube consists of a $17 \mathrm{~m}$ long $300 \mathrm{~mm}$ inner diameter tube, a transition piece to allow for the upstream filling of the tube, an axisymmetric nozzle, a diaphragm station (located either just upstream of the throat or downstream of the test section), and a dump tank.

Before a run, the tube is filled with the test gas at a pressure of up to $700 \mathrm{kPa}$ and the dump tank is evacuated. To start the run, the diaphragm is ruptured, thus causing an expansion wave to propagate through the nozzle and into the tube. When the diaphragm is downstream of the test section, it is ruptured in a controlled way using a cutting device. In the upstream position, the diaphragm is ruptured by creating a sufficient pressure difference across it because any cutting

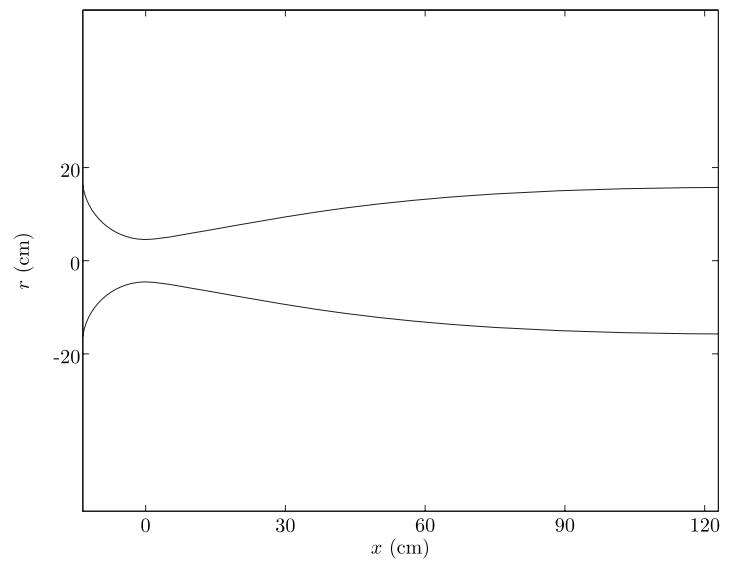

FIG. 2. Mach 4.0 nozzle contour designed by Korte of NASA Langley Research Center.

device upstream of the nozzle would disturb the flow excessively. During the time it takes for the expansion wave to travel to the end of the tube and for the reflected wave to return to the nozzle, the reservoir conditions for the nozzle flow are near uniform, thus giving a constant condition test time of about $100 \mathrm{~ms}$.

A Mach number of 4 was chosen because it is necessary to operate at a Mach number for which the dual-solution domain has a shock angle range that is not too small. Also, to avoid having to heat the air in the tube, it is necessary to operate at a Mach number no higher than 4 to avoid condensation of the test gas in the nozzle expansion.

At this Mach number it was better to make the nozzle axisymmetric rather than rectangular. In part, this is to eliminate the unavoidable secondary flow in the corners, and the complications of a circular to rectangular transition piece. Since the test rhombus is quite slender at Mach 4, a considerable portion of the test rhombus lies downstream of the nozzle exit so that a useful portion of the test section can be fitted with flat windows set back from the nozzle edge, so no re-entrant corner is visible to the flow.

The contours for the Mach 4.0 nozzle, shown in Fig. 2, and the original Mach 2.3 nozzle were designed by Korte of NASA Langley Research Center. The Mach 2.3 nozzle has been calibrated by using the weak Mach wave technique. ${ }^{7}$

\section{B. Mach 4 nozzle}

Since the expansion takes only about $100 \mathrm{~ms}$ to travel from the nozzle to the end of the tube and back, any time needed to establish steady supersonic flow reduces the test time. It is therefore important that the nozzle design allows for a fast startup process. Computations made assuming an infinite dump tank and a diaphragm downstream of the nozzle are shown as a time sequence in Fig. 3. These computations were performed using an Euler solver that is part of the Amrita system. ${ }^{8}$ The image shows the initial condition at $t=0 \mathrm{~ms}$, when the diaphragm separates the high pressure gas in the tube and nozzle from the low pressure region in the dump tank. At $t=4 \mathrm{~ms}$ the shock generated by the diaphragm rupture has traveled downstream into the dump tank and an expansion wave has propagated upstream through the 

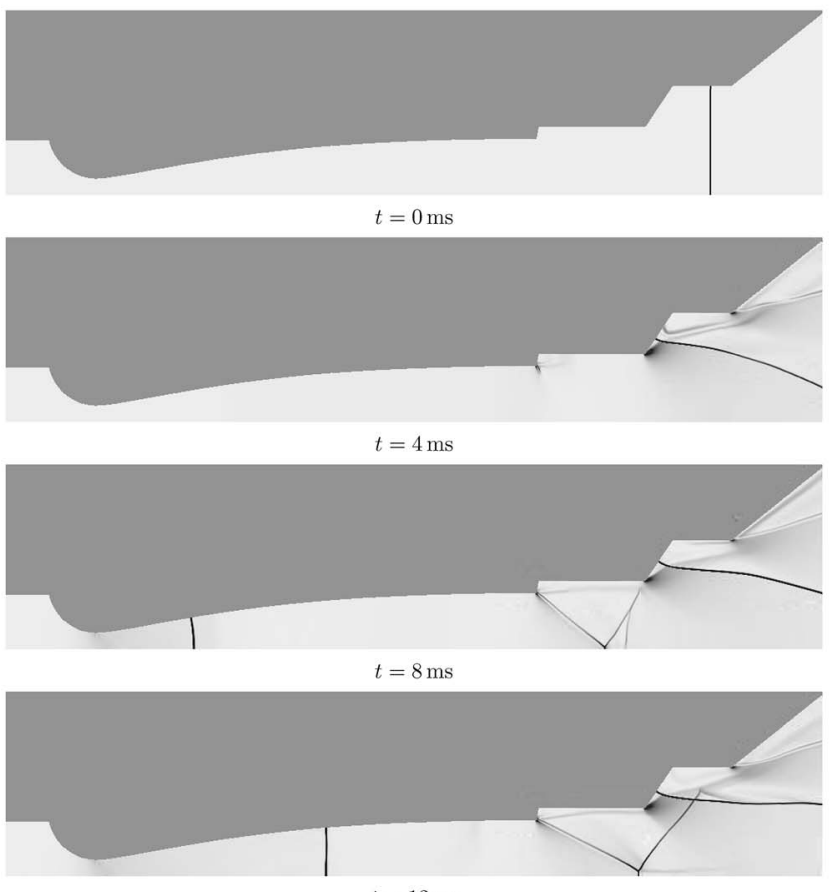

$t=12 \mathrm{~ms}$
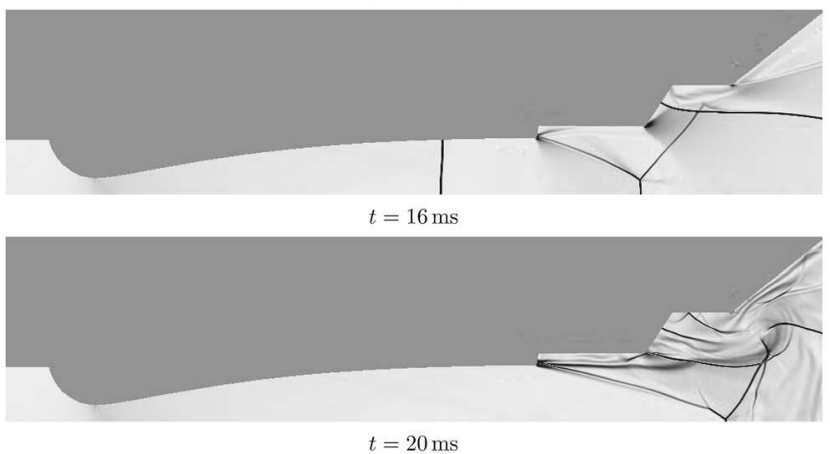

FIG. 3. Startup process of the Mach 4 nozzle computed using Amrita. The frames are quasi-Schlieren images of the flow at different times in the startup process assuming an infinite dump tank.

nozzle. At $t=8 \mathrm{~ms}$ the expansion wave has partially reflected from the throat and formed an upstream facing shock that is being convected downstream because its propagation speed relative to the gas is smaller than the speed of that gas. This shock is seen just downstream of the throat. At $t=12 \mathrm{~ms}$ and $t=16 \mathrm{~ms}$ the reflected shock continues to be washed downstream through the nozzle. At $t=20 \mathrm{~ms}$ the reflected shock has moved downstream past the first expansion characteristic from the end of the nozzle, and steady flow in the test section is established. Thus, one may expect the startup to take $20 \mathrm{~ms}$.

Early experiments showed that it was not possible to start the flow properly when using a downstream diaphragm. A Schlieren image of the actual flow taken halfway through the test time is shown in Fig. 4. The maximum steady-flow time achieved with this configuration was no more than 20 ms. Amrita simulations were conducted to understand the problem. In particular, the problem was modeled with a finite dump tank to understand the importance of the back pressure as well as the shock generated when the diaphragm is ruptured.

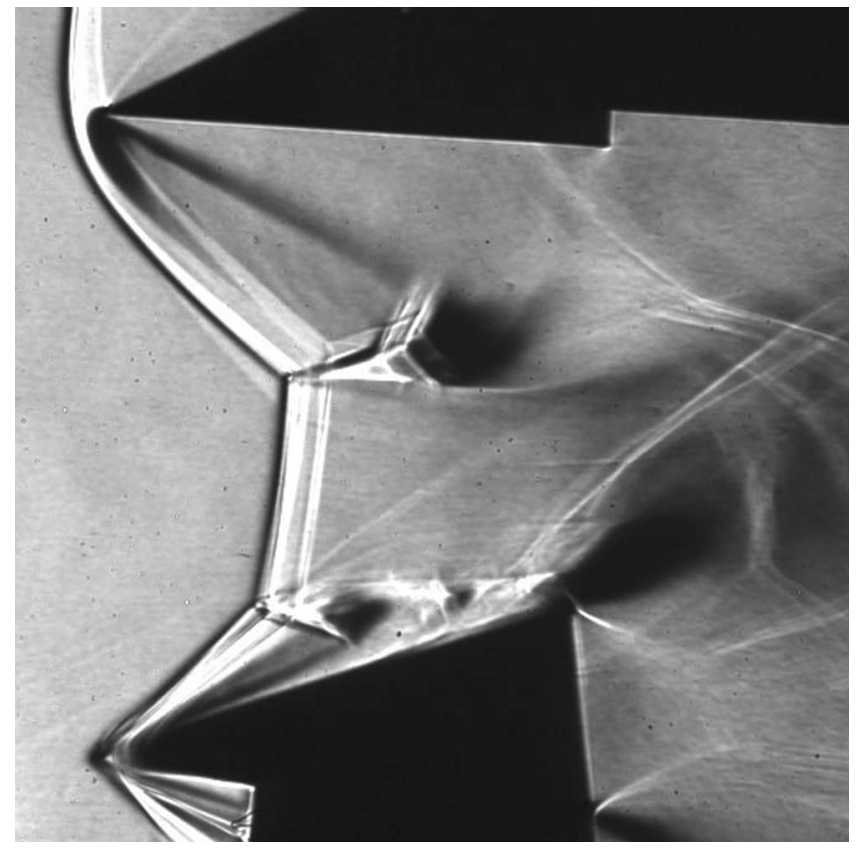

FIG. 4. Experimental flow at approximately $50 \mathrm{~ms}$ into the test time showing unstart.

\section{Dump tank}

In previous experiments using the Ludwieg tube with a Mach 2.3 nozzle the shock that propagated into the dump tank and reflected back posed no problems. However, with the Mach 4.0 nozzle this reflected shock returned to the test section earlier and disrupted the flow. Computations using Amrita, with the dump tank fully modeled confirmed this problem. An extensive study of possible solutions was made to find a way both to reduce the strength of this reflected shock and to prevent it from re-entering the test section. Figure 5 is the result of a simulation showing the reflected shock from the dump tank inside the test section.

In the simulations, the addition of a baffle and a tube extension inside the dump tank, at least computationally, solved the problems associated with the reflected startup shock. Figure 6 shows the result of a computation with the addition of the baffle and the tube extension. The flow inside the test section remains steady throughout the test time. The design and placement of the baffle and extension tube are shown in Fig. 7. The baffle is supported by three Unistruts attached to the dump tank flange. There is also a $505 \mathrm{~mm}$ outer diameter tube placed between the three Unistruts and attached to the dump tank flange.

A computational sensitivity study with respect to the location of the baffle showed that the baffle had to be placed within about one foot of the design location, which, given

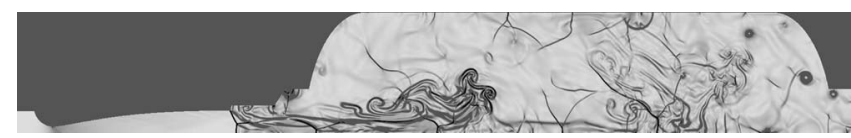

FIG. 5. Simulation showing the unstart of the nozzle, $51.8 \mathrm{~ms}$ after the rupturing of the diaphragm, as a result of the reflected shock from the end of the dump tank. 


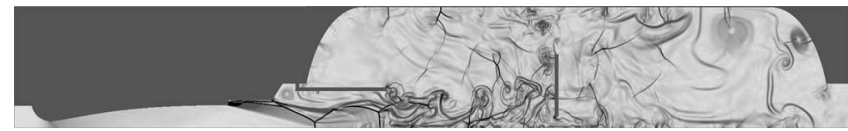

FIG. 6. Simulation showing undisturbed flow in the test section, $51.7 \mathrm{~ms}$ after diaphragm rupture. The influence of the reflected shock has been kept away from the test section as the result of the addition of the baffle and tube extension

the limits in fully modeling the physics of the problem, was cause for concern. Experiments with these modifications installed in the dump tank again resulted in flow unstart, very similar to what is seen in Fig. 4. The more drastic modification of moving the diaphragm upstream of the throat was then considered.

\section{Upstream diaphragm station}

Further computations, with the diaphragm moved just upstream of the converging nozzle section showed no problems with flow unstart and also produced a flow start time of only $3 \mathrm{~ms}$, as opposed to the $20 \mathrm{~ms}$ required with the downstream diaphragm. This successful start is shown in Figs. 8 and 9 .

Unfortunately, there are significant drawbacks to having the diaphragm upstream of the test section. Specifically, the ruptured diaphragm will cause disturbances due to its presence as well as due to the production of small pieces of debris. Experiments conducted using an upstream Mylar diaphragm of 5 mil thickness confirmed the fast startup process, and unstart of the flow was not experienced until after the reflected expansion wave from the tube returned to the test section. The experimental run time using the upstream diaphragm was nearly the full $100 \mathrm{~ms}$.

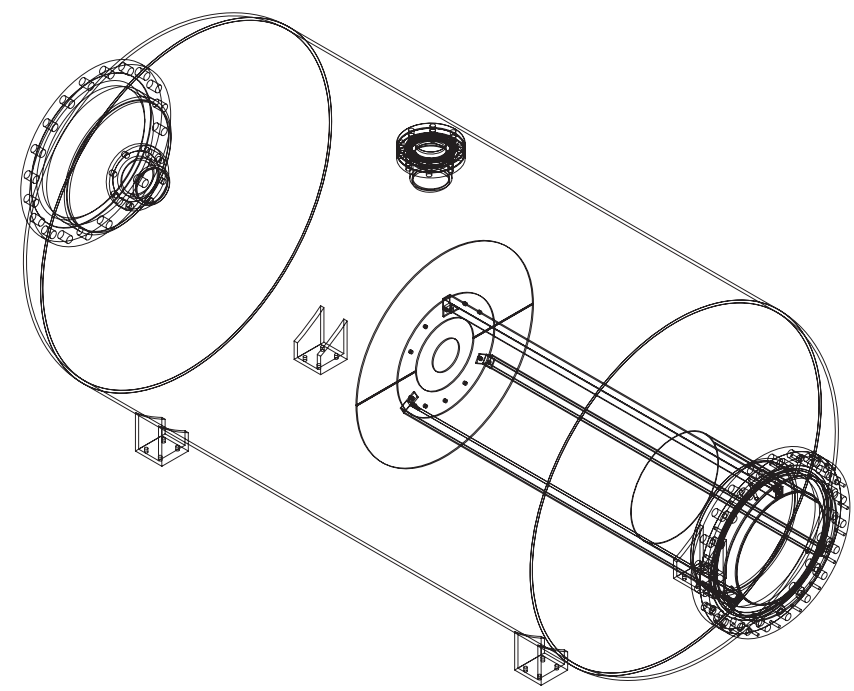

FIG. 7. Dump tank with modifications including the addition of a baffle and a tube extension. The baffle is connected to the dump tank with a series of unistruts.

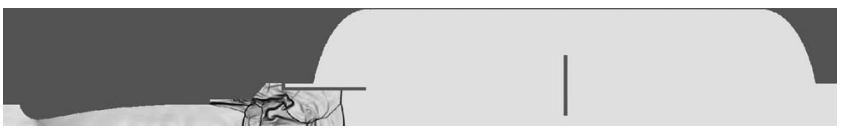

FIG. 8. Simulation showing the starting of the nozzle, $2.7 \mathrm{~ms}$ after the rupturing of the diaphragm located just upstream of the converging section of the nozzle. The left boundary condition, just upstream of the throat, is extrapolated, while the right boundary condition, at the end of the dump tank, and the outer wall of the dump tank are reflective.

\section{E. Adjustable wedge model}

A double wedge model was constructed with the lower wedge being fixed at an angle of $23.6^{\circ}$ and the upper wedge being adjustable. This asymmetric wedge configuration, like that of Sudani et al., ${ }^{6}$ was chosen for experimental simplicity. The upper wedge angle is controlled using a rotary servomotor with a 5:1 gear ratio. The assembly of the adjustable wedge is shown in Fig. 10. The wedge is supported by two vertical posts, and its motion is controlled by a connecting rod, which is connected to the motor.

\section{Motor and gear box}

A Parker electromechanical BE342KR brushless servomotor with a Bayside PX34-005 inline planetary gear head with a 5:1 gear ratio is used. The motor itself provides a maximum speed of $5000 \mathrm{rpm}$ and maximum torque $3.12 \mathrm{~N} \mathrm{~m}$. This provides a maximum speed of $1000 \mathrm{rpm}$ and a maximum torque of $15.6 \mathrm{~N} \mathrm{~m}$ after the gear head. It is estimated that aerodynamic forces on the wedge generate a torque on the wedge not exceeding $7 \mathrm{~N} \mathrm{~m}$, in part because the pivot point is placed close to the center of pressure. In most cases, since the initial tube pressure in the present experiments is less than the maximum tube pressure, the actual torque on the wedge will be significantly smaller.

The motor is controlled by a Parker Compax 3 programmable servo positioner. The motor is programmed to follow a "S-curve" in order to minimize jerk.

\section{F. High speed Schlieren photography}

The Ludwieg tube is equipped with a high-speed Schlieren system. The primary component of the system is a Visible Solutions Phantom v7.1 camera. At the full resolution of $800 \times 600$ pixels the camera has a frame rate of 4800 fps. The frame rate increases to $8300 \mathrm{fps}$ at $512 \times 512$ pixels and to 27000 fps at $256 \times 256$ pixels. A key feature of the camera is the ability for the user to specify the exact aspect ratio and resolution. By doing this no pixels are wasted on uninteresting parts of the flow. This results in higher resolution and higher speed compared to that of a fixed aspect ratio camera.

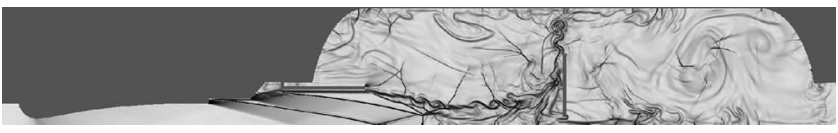

FIG. 9. Simulation showing the correctly started flow $50.1 \mathrm{~ms}$ after the rupturing of the diaphragm located just upstream of the converging section of the nozzle. 


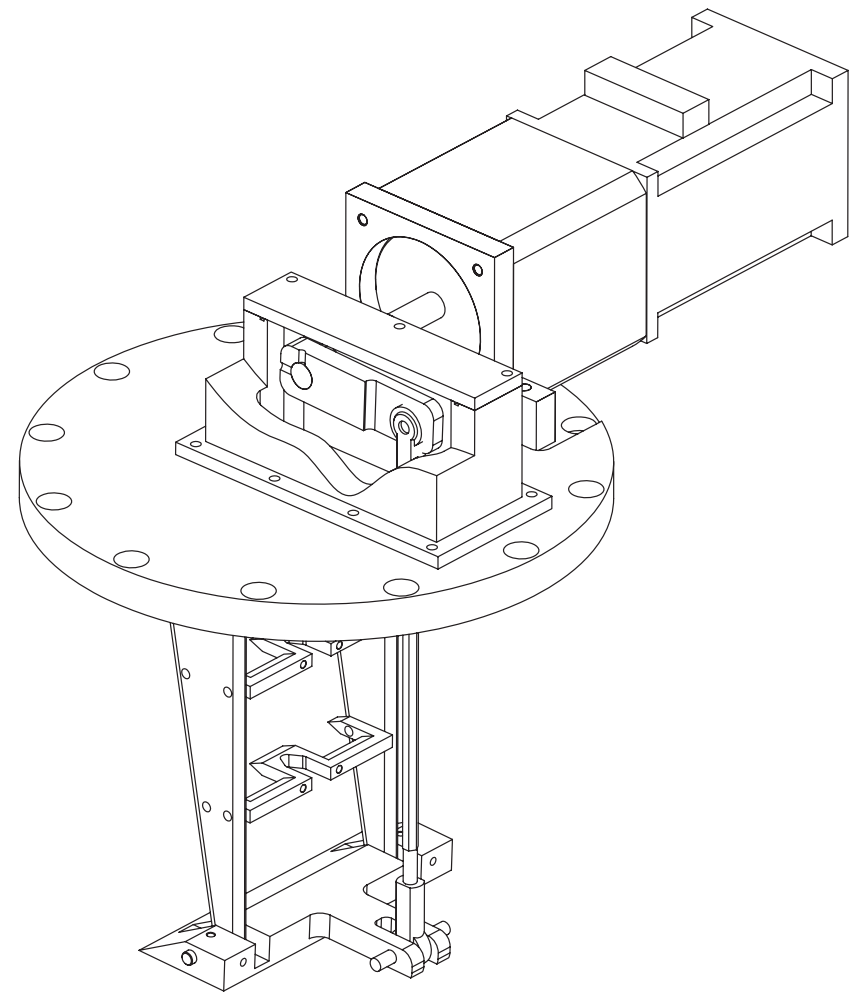

FIG. 10. Wedge assembly consisting of a wedge, a connecting rod, a support structure, a motor, and a gear head.

The light source for the system is an Oriel 66181 with corresponding power supply. The unit has a $1000 \mathrm{~W}$ quartz tungsten halogen lamp. The remainder of the Schlieren setup is a standard Z-fold configuration.

\section{EXPERIMENTAL HYSTERESIS}

Experimental realization of hysteresis in Mach reflection is a qualitative measure of the quietness of the flow. Specifically, the quieter the flow, the closer one can approach the upper end of the dual-solution domain while maintaining regular reflection.

In order to demonstrate hysteresis, the upper adjustable wedge was set so that the shocks were initially below the von Neumann condition, and therefore only regular reflection was possible. The adjustable wedge angle was then increased about $15^{\circ}$ over $40 \mathrm{~ms}$ (670 characteristic flow times) and subsequently decreased about $15^{\circ}$ over $40 \mathrm{~ms}$ (670 characteristic flow times). The effect of asymmetry on the von Neumann and detachment conditions was discussed by Mouton, ${ }^{2}$ see also Sudani et al. ${ }^{6}$ This initial configuration is shown in Fig. 11(a).

The angle of the upper wedge was slowly increased to bring the shocks into the dual-solution domain while maintaining regular reflection. Figure 11(b) shows regular reflection inside the dual-solution domain, and illustrates the highest angles obtainable in the Ludwieg tube, without transitioning to the Mach reflection. It should be pointed out that both Ivanov and Sudani were able to penetrate deeper into the dual-solution domain than our experiments. (a)

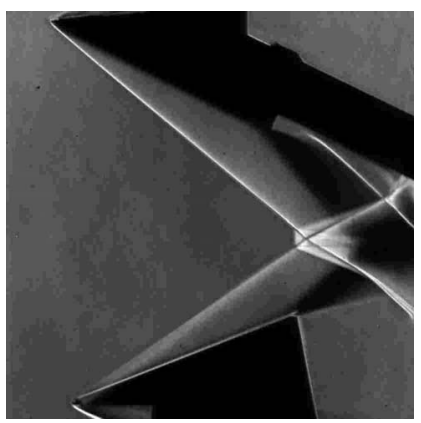

(c)

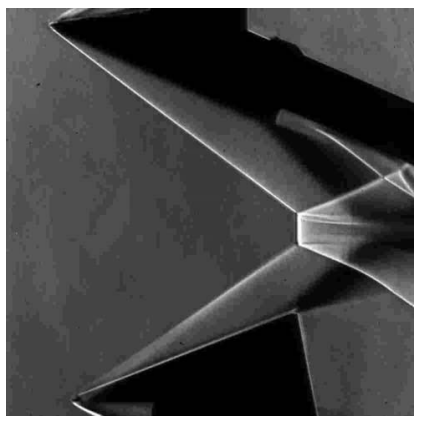

(e)

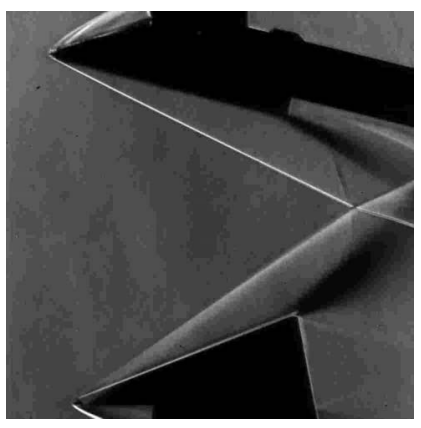

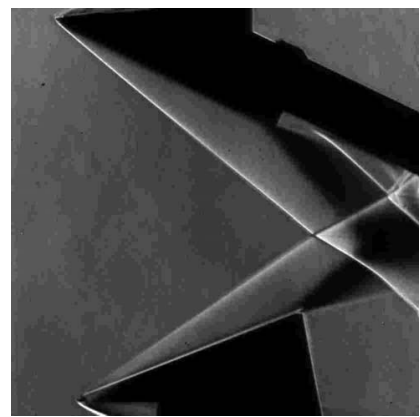

(b)

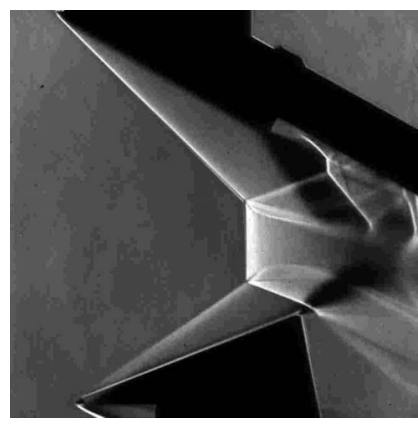

(d)

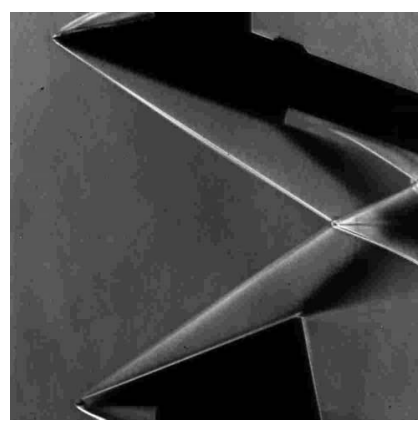

(f)
FIG. 11. Demonstration of the hysteresis phenomenon in the Ludwieg tube. The initial wedge angles were set so that only regular reflection was possible and then the upper wedge angle was increased into the dual-solution domain. The upper wedge angle was then increased further so that tunnel disturbances would cause transition to the Mach reflection. The upper wedge angle was then decreased to below the von Neumann condition. $M=4.0$, $\alpha_{\text {lower }}=33.9^{\circ}$. The upper wedge angles, $\alpha_{\text {upper }}$, are $25.9^{\circ}, 39.8^{\circ}, 39.9^{\circ}, 45.0^{\circ}$, $37.9^{\circ}$, and $33.0^{\circ}$ in parts (a), (b), (c), (d), (e), and (f), respectively. The times, $t$, are 13.4, 49.0, 49.3, 64.6, 81.7, and $85.7 \mathrm{~ms}$ in parts (a), (b), (c), (d), (e), and (f), respectively.

A further increase in the upper wedge angle will cause a transition to the Mach reflection inside the dual-solution domain. Figure 11(c) shows a Schlieren image just after transition to the Mach reflection has begun. The image shows the three dimensionality of the Mach stem, with both regular reflection and Mach reflection being visible in the image.

As the upper wedge angle continues to increase, the Mach stem grows in size. This larger Mach stem, corresponding to the high incident shock angle from the upper adjustable wedge, is shown in Fig. 11(d).

With Mach reflection established, the wedge angle can be decreased. Figure 11(e) shows Mach reflection inside the dual-solution domain, at an angle smaller than in Fig. 11(b), where there was regular reflection. 
As the upper wedge angle is decreased further, the Mach stem height also decreases. The Mach stem height will eventually decrease to zero at the von Neumann condition and the reflection will return to regular reflection, as is seen in Fig. 11(f). The angles at which the transition back to regular reflection occurs agrees very well with the theoretical von Neumann condition of $\alpha_{\text {lower }}=33.9^{\circ}$ and $\alpha_{\text {upper }}=33.0^{\circ}$.

\section{ENERGY DEPOSITION}

Besides transitioning from regular reflection to the Mach reflection by increasing the wedge angle to a sufficient extent that the tunnel disturbances cause transition, it is also possible to introduce a disturbance to cause this transition to happen. Sudani et al. ${ }^{6}$ showed that various small disturbances can trip the flow from regular reflection to the Mach reflection in the dual-solution domain. Numerical studies by Khotyanovsky et al. ${ }^{9}$ showed that energy deposition can cause transition from regular to Mach reflection. In addition, they showed that with enough mesh refinement, transition from Mach to regular reflection by means of energy deposition is not possible.

A numerical study by the current authors of a dense lump of gas impacting the wedge suggested that it is the shock formed by the impact itself that is the key in determining whether transition from regular reflection to the Mach reflection occurs. ${ }^{2}$ Because of this, it is useful to study the simplified case of energy deposition directly on the wedge surface. In the two-dimensional case, the shock formed by this energy deposition will be cylindrical, and while it is strong it will behave according to blast wave equation for strong shocks, as obtained by Sedov. ${ }^{10}$

\section{A. Minimum energy requirement}

A minimum required energy can be constructed by the fact that the blast wave must reach the leading shock in order to have an influence on whether or not transition from regular reflection to the Mach reflection occurs. In order to determine if the blast wave will reach the leading shock, we must know the trajectory of the shock as a function of time and of the amount of energy deposited. This analysis neglects the effects of the expansion wave off the aft wedge corner. We can write the radius of the blast wave, $R_{\mathrm{s}}$, as a function of the time, $t$, the energy deposited, $E_{0}$, and the density and pressure into which the blast wave is propagating, $\rho_{1}$ and $P_{1}$, respectively. An analytical solution, assuming a strong shock, is given by Sedov. ${ }^{10}$ For nonstrong shocks, the solution was computed using an Euler solver developed by N. F. Ponchaut (private communication). The matching between the analytic and computed solutions is shown in Fig. 12.

Knowing the complete solution it is possible to find the energy, $E_{0}$, required for the blast wave to reach the leading oblique shock. The blast wave will convect downstream with the mean flow in region 1, as shown in Fig. 13. The limiting case is defined as the energy required for the blast wave to just reach the reflection point. Based on Fig. 13, we can write the minimum distance, $l_{\min }$, between the blast wave and the reflection point as

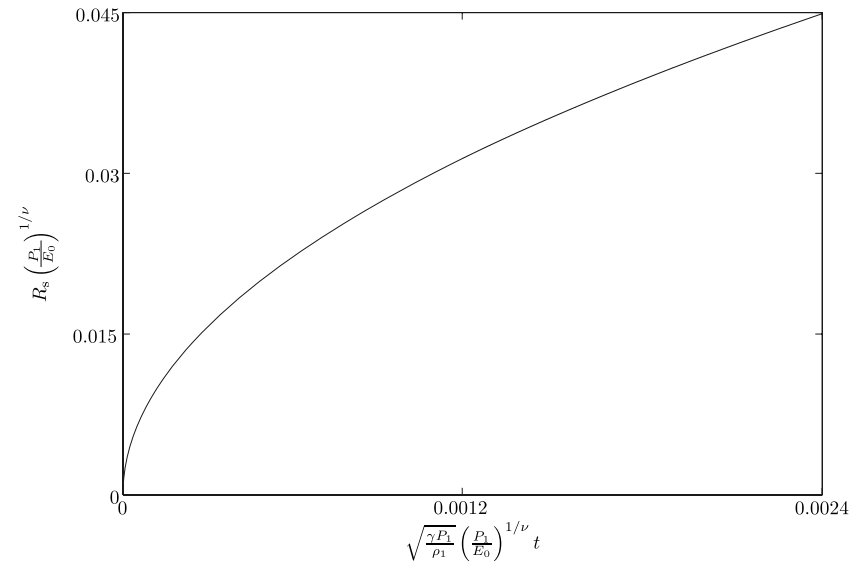

FIG. 12. The graph to the left of 0.001164 is from Sedov's exact solution for strong shocks, and the graph to the right of 0.001164 is the Euler solution. Very good continuation of Sedov's solution is seen. $\nu=2$ and $\gamma=1$.4.

$$
l_{\min }=\sqrt{\left(l_{1}-u_{1} t\right)^{2}+l_{2}^{2}}-R_{\mathrm{s}},
$$

where $u_{1}$ is the flow speed in region 1 , and

$$
\begin{aligned}
& l_{1}=G \cos \theta_{1}\left(\cot \alpha-\cot \theta_{1}\right)+d \csc \theta_{1}, \\
& l_{2}=G \csc \alpha \sin \left(\alpha-\theta_{1}\right) .
\end{aligned}
$$

The minimum distance, $l_{\min }$, will be positive when the blast wave has not reached the reflection point, and will be negative when the blast wave has expanded beyond the reflection point.

The conditions for the minimum energy required for the blast wave to reach the reflection point is

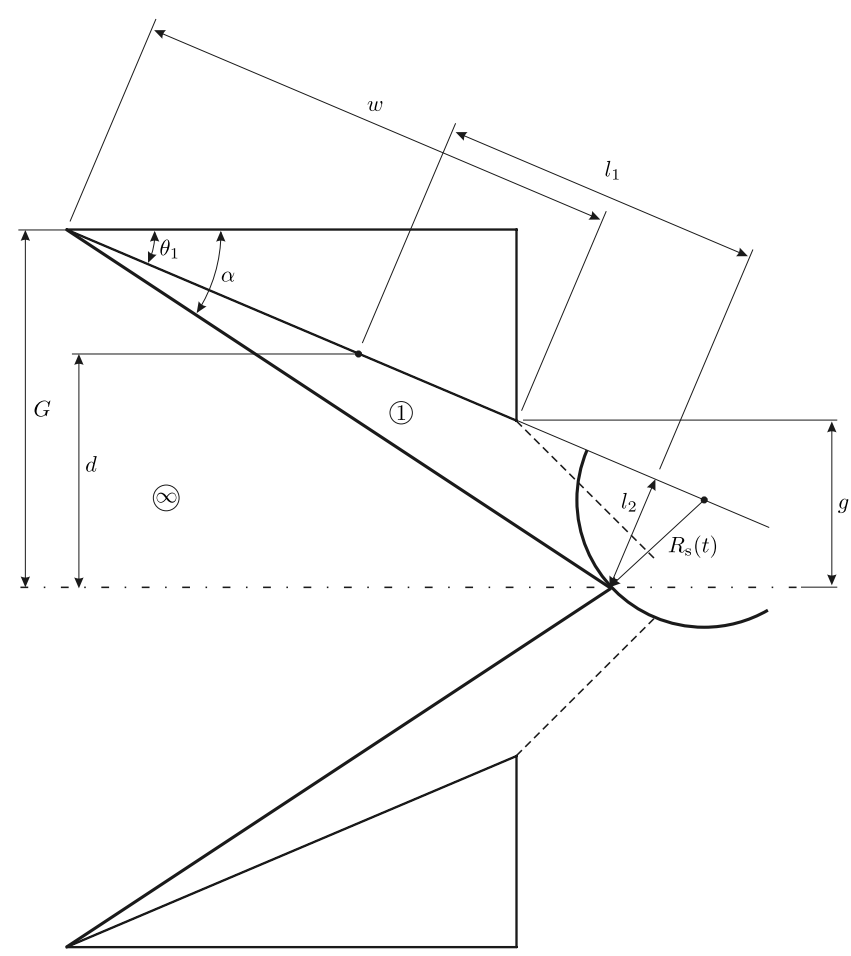

FIG. 13. Flow setup considering energy deposition along the wedge surface showing the blast wave just reaching the point of reflection. 


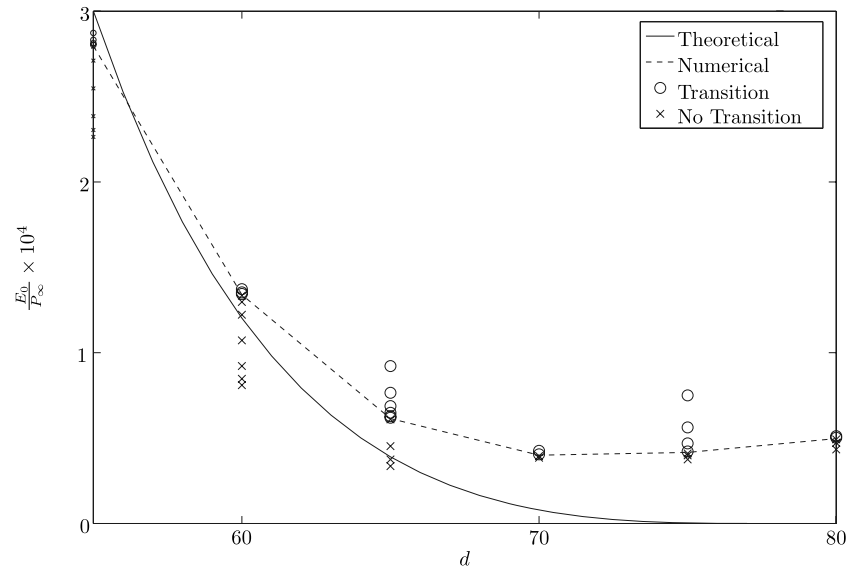

FIG. 14. Energy, $E_{0}$, required for transition from regular to the Mach reflection to occur. The solid line represents the theoretical minimum energy curve as discussed earlier in this section, the dashed line represents the curve fit to the computational results. The $\times$ indicates that transition did not occur at the given condition; whereas, the $\bigcirc$ indicates that transition did occur. Computations done for $M=4, \nu=2, \gamma=1.4, G=120$, and wedge angle, $\theta_{1}=25^{\circ}$.

$$
\begin{aligned}
& \left.R_{\mathrm{s}}\right|_{t=t_{\mathrm{s}}}=\sqrt{\left(l_{1}-u_{1} t_{\mathrm{s}}\right)^{2}+l_{2}^{2}}, \\
& U_{\mathrm{s} \mid t=t_{\mathrm{s}}}=\left.\frac{d R_{\mathrm{S}}}{d t}\right|_{t=t_{\mathrm{s}}}=\frac{u_{1}\left(u_{1} t_{\mathrm{s}}-l_{1}\right)}{\sqrt{\left(l_{1}-u_{1} t_{\mathrm{s}}\right)^{2}+l_{2}^{2}}} .
\end{aligned}
$$

This condition states that in the minimum energy case, the blast wave will reach the reflection point at time $t_{\mathrm{s}}$ and then retreat from the reflection point. When the blast wave originates sufficiently far from the reflection point even a Mach wave can reach the reflection point, in this case the minimum energy required is zero. This occurs when

$$
\frac{l_{1}}{l_{2}} \sqrt{1+\left(\frac{l_{2}}{l_{1}}\right)^{2}} \geq M_{1} \text {. }
$$

Using Amrita ${ }^{8}$ and an iterative technique, the energy required to cause transition can be calculated. The Euler solver was set to run a case and then output whether or not transition to the Mach reflection occurred. A standard bisection method was then employed using this information. To increase the speed of the routine, previous results were used to calculate a first guess when a different wedge angle was used. The results of 47 separate cases are plotted in Fig. 14.

\section{B. Experimental transition by energy deposition}

In order to enter into the dual-solution domain with regular reflection, the upper adjustable wedge is first set to an angle below the von Neumann condition, such that only regular reflection is possible (as seen in Fig. 15). The wedge is then slowly rotated into the dual-solution domain (as seen in Fig. 16), while remaining below the point where tunnel disturbances would cause transition. The vertical black line seen in the images is used as a reference, to enable accurate shock angle measurement.

Visualization of the blast wave created by the energy deposition was done by examining the laser deposition of energy without flow at atmospheric conditions. Figure 17

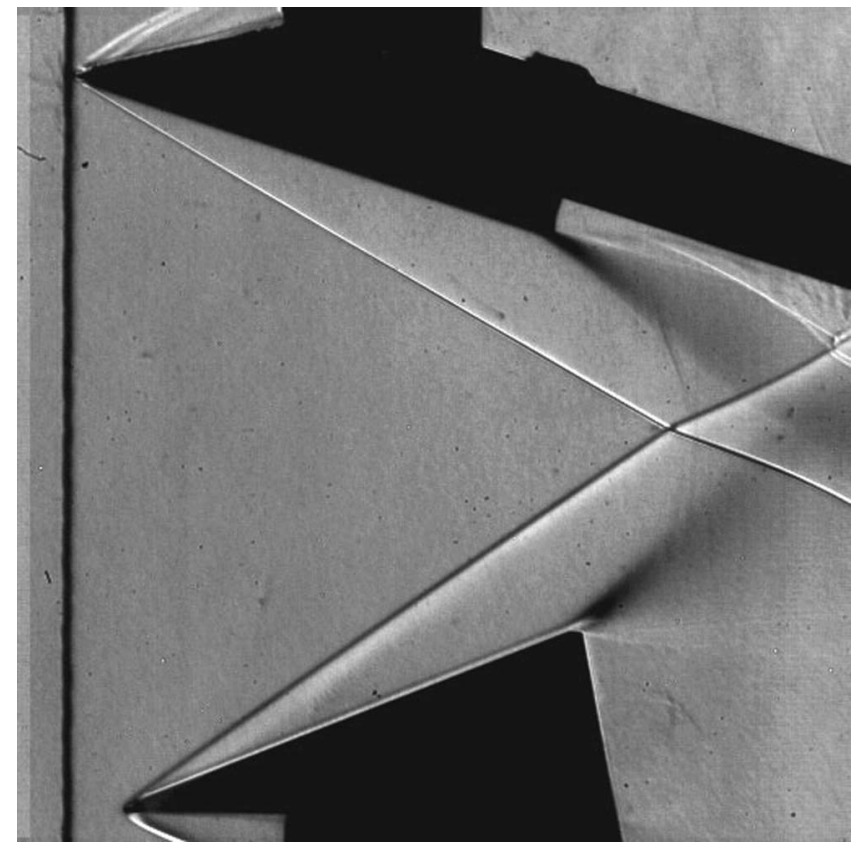

FIG. 15. Initial shock configuration below the von Neumann condition. Only regular reflection is possible. $M=4, \alpha_{\text {lower }}=33.8^{\circ}$, and $\alpha_{\text {upper }}=29.9^{\circ}$.

shows Schlieren images after deposition of energy on the lower wedge in which the resulting blast wave may be seen. Unfortunately, the gas density during the flow is too low to be able to detect the blast wave from Schlieren images. The blast wave from the energy deposition brings about a disturbance, which is seen in Fig. 18 as an outward bulging of the leading shock.

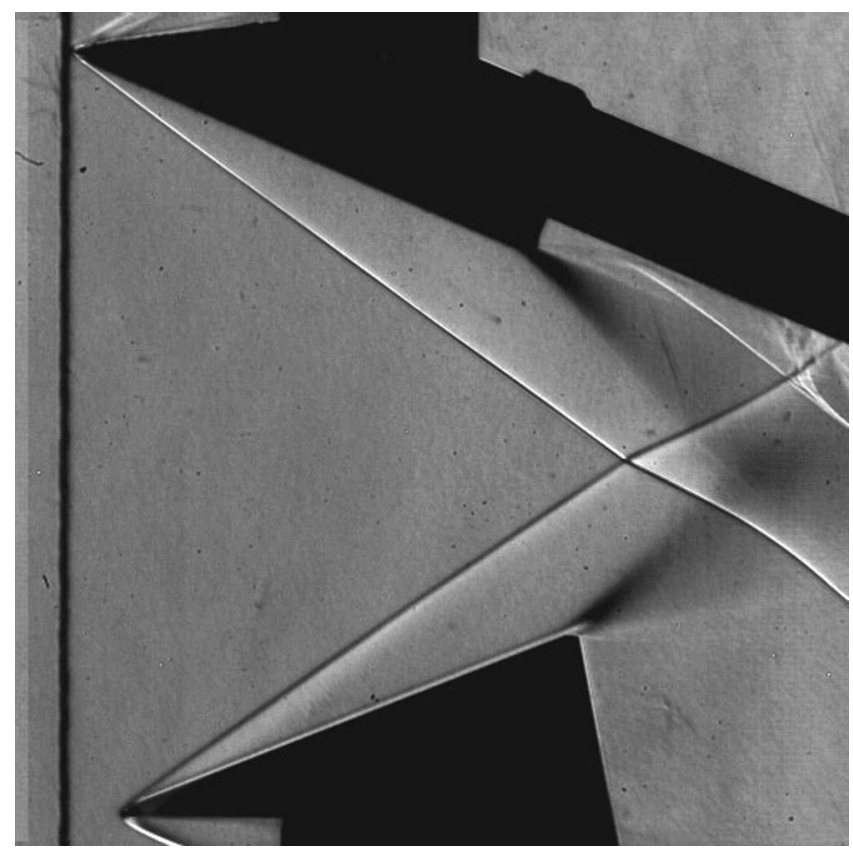

FIG. 16. Shock configuration before laser energy is deposited onto the lower wedge. Both regular reflection and the Mach reflection are possible. $M=4$, $\alpha_{\text {lower }}=33.8^{\circ}$, and $\alpha_{\text {upper }}=36.0^{\circ}$. 


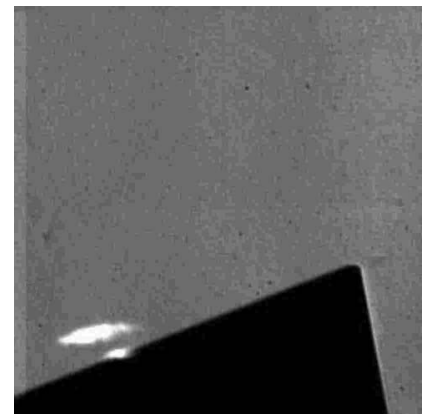

(a)

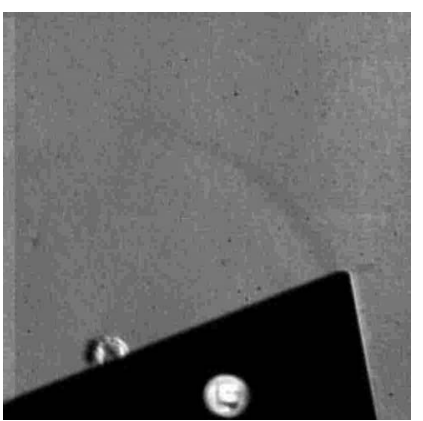

(c)

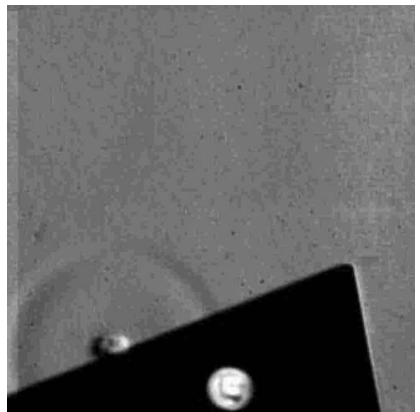

(b)

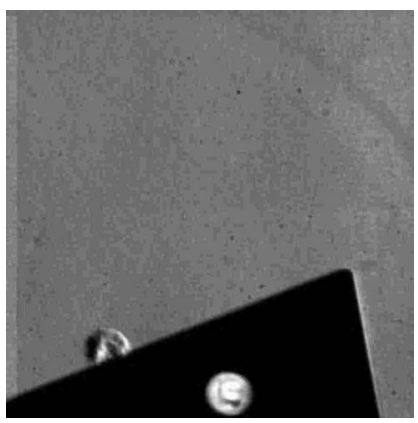

(d)
FIG. 17. Blast wave resulting from the deposition of energy on the lower wedge using a laser. The exposure of each image was $3 \mu$ s, with $38 \mu \mathrm{s}$ between exposures. The circular light seen inside the lower wedge in images (b), (c), and (d) is used to indicate that the laser has fired.

\section{Energy deposition location}

Figure 14 shows the importance of energy deposition location. By focusing the laser at various positions along the wedge it was found that for positions on the wedge close to the centerline, i.e., small $d$, transition from regular reflection

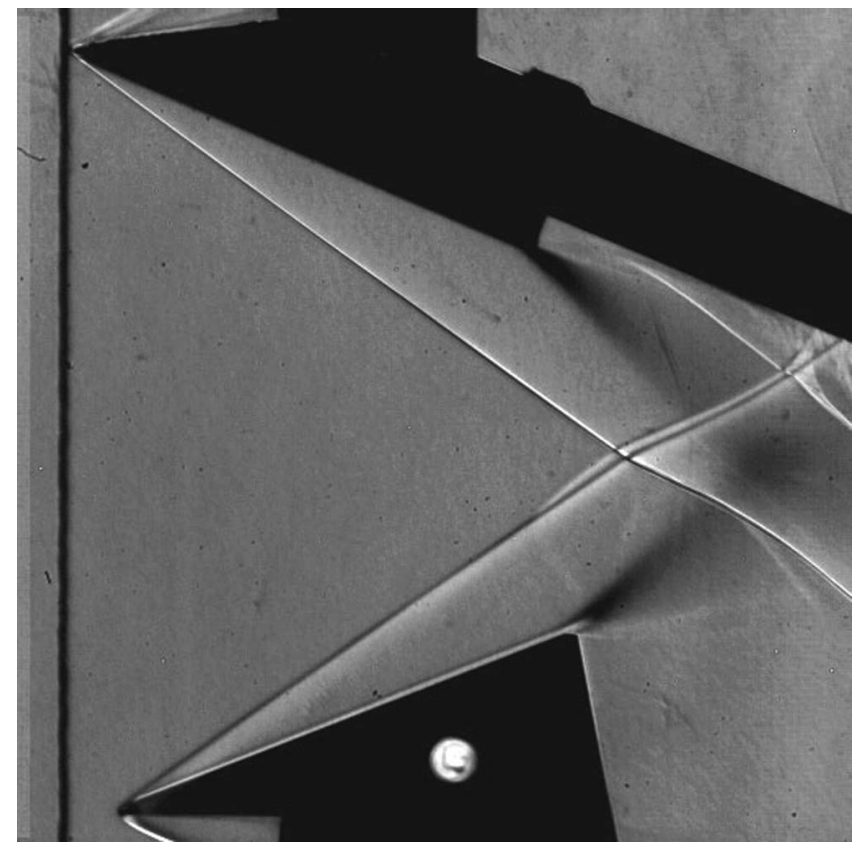

FIG. 18. The leading shock is disturbed in the region of the reflection due to the laser energy, which was previously deposited. Transition to Mach reflection will immediately follow. $M=4, \alpha_{\text {lower }}=34.5^{\circ}$, and $\alpha_{\text {upper }}=35.8^{\circ}$.
TABLE I. Summary of transition for various energy deposition locations.

\begin{tabular}{cc}
\hline \hline Location $(d / G)$ & Transition from RR $\rightarrow \mathrm{MR}$ \\
\hline 0.553 & Yes \\
0.594 & Yes \\
0.645 & Yes \\
0.689 & Yes \\
0.752 & Yes \\
0.811 & No \\
0.867 & No \\
\hline
\end{tabular}

to the Mach reflection always occurred. However, it was observed that there was a distance from the centerline beyond which transition would not occur. This is because, for large $d$, the blast wave has a large distance to travel before reaching the reflection point, and hence becomes too weak to cause transition once it does reach the reflection point. The fact that transition occurs for small $d$ means that the energy is sufficient for the blast wave to reach the reflection point in these cases. This is consistent with the results presented in Sec. IV A, which show that if $d$ is small and the blast wave is strong enough to reach the reflection point, transition will occur. A summary of the seven energy location experiments is given in Table I. For the asymmetric case, $G$ is taken to be half the vertical distance between the leading edges of the two wedges, and $d$ is measured from this dividing line.

These results are shown graphically in Fig. 19, where the energy deposition points that caused transition are marked with a $\bigcirc$, and deposition points that did not lead to transition are denoted with $\mathrm{a} \times$.

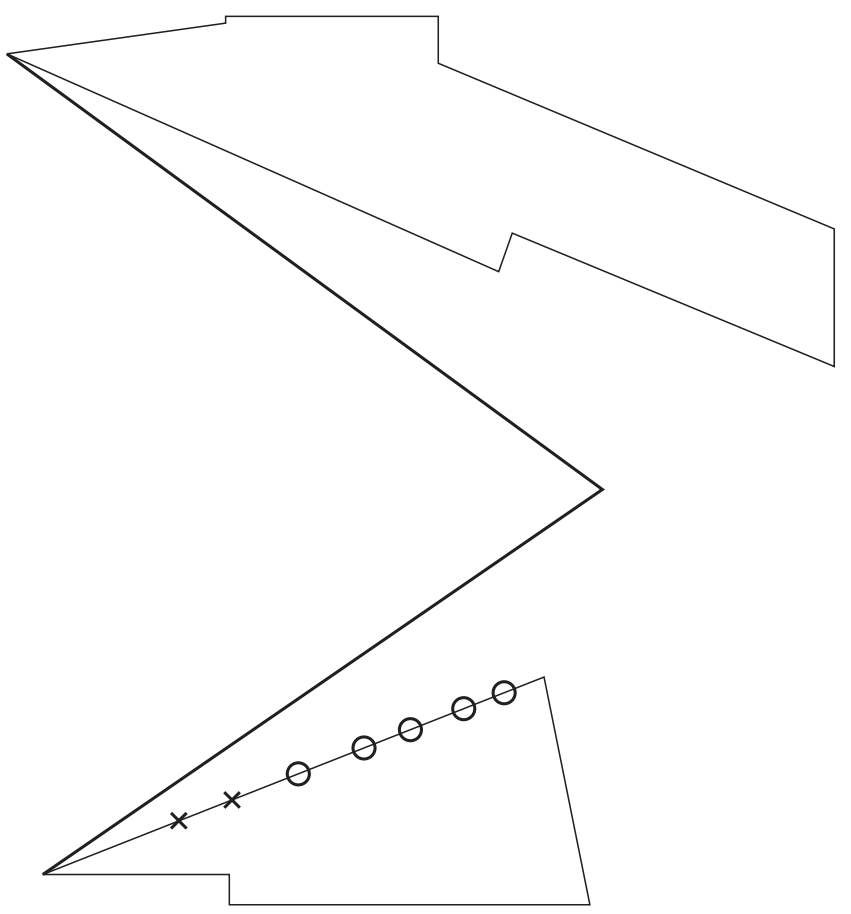

FIG. 19. Energy deposition points on the lower wedge. Deposition points which lead to transition are denoted with a $\bigcirc$; whereas, deposition points that did not lead to transition are denoted with a $\times$. 


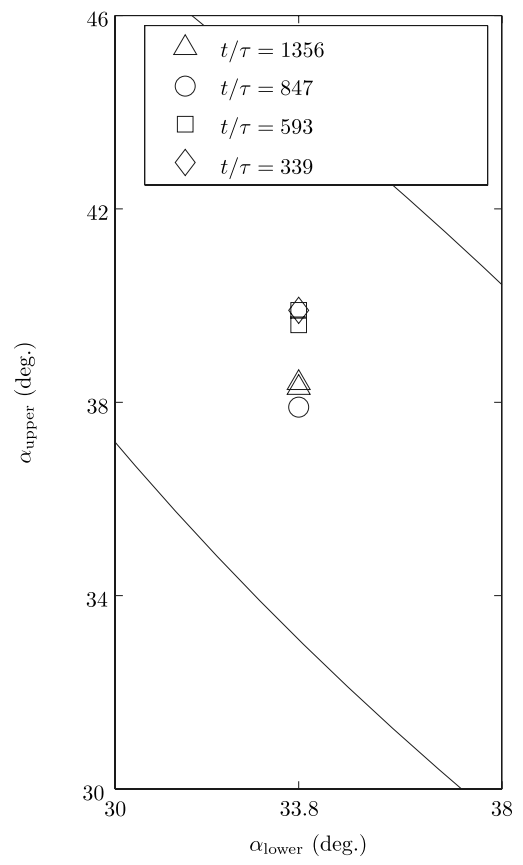

FIG. 20. Effect of wedge rotation speed on tripping due to tunnel disturbances. As the wedge is rotated faster, higher shock angles are obtained while maintaining regular reflection. The lower curve is the von Neumann condition and the upper curve is the detachment condition. Transition occurs approximately halfway into the dual-solution domain.

\section{TUNNEL DISTURBANCES}

Disturbances inherent to the test facility can also cause the flow to trip from regular to Mach reflection. To explore this, the wedge was moved at different speeds into the dualsolution domain without any artificially added disturbances. The angle at which transition occurred was then measured. Since the test time is limited to about $100 \mathrm{~ms}$ of flow time, the wedge must be moved relatively quickly. One might expect that as the wedge is moved faster, transition would occur earlier; however, it was found that at higher speeds it was possible to maintain regular reflection up to higher shock angles.

The characteristic flow time for the lower wedge is

$$
\tau=\frac{w}{u_{1}},
$$

where $u_{1}$ is the speed behind the incident shock of the lower wedge. For the current experiments, $w=50.8 \mathrm{~mm}$ and $u_{1}=983 \mathrm{~m} / \mathrm{s}$, which give a characteristic flow time, $\tau$, of $59 \mu \mathrm{s}$. Figure 20 shows the effect of wedge rotation speed on the transition point, where $t$ is the time to rotate the upper wedge $10^{\circ}$, starting from an initial angle of $20^{\circ}$. Transition from regular to the Mach reflection occurs approximately halfway into the dual-solution domain.

Most often when transition occurs due to tunnel disturbances no visible disturbance is noticed. However, in a few cases, a significant disturbance just before tripping from regular reflection to Mach reflection is observed. Figure 21 shows a small piece of dust, possibly a piece of the diaphragm, traveling downstream near the centerline. Because

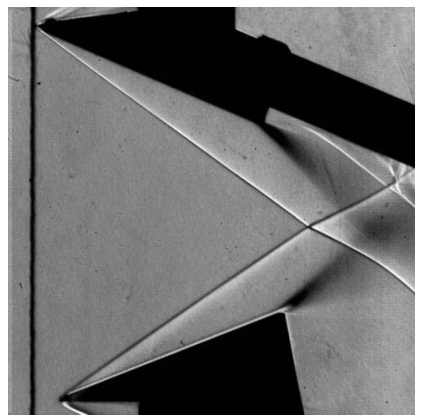

(a)

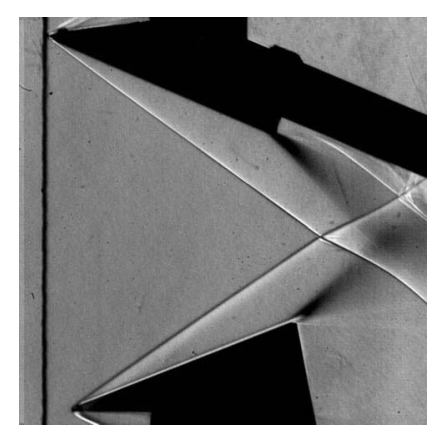

(c)

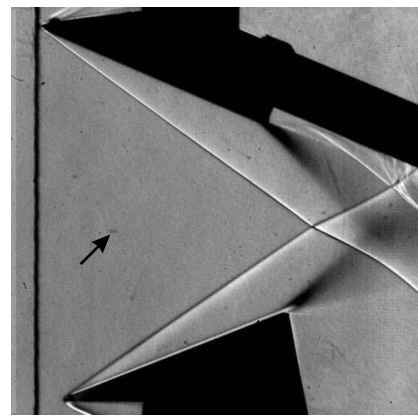

(b)

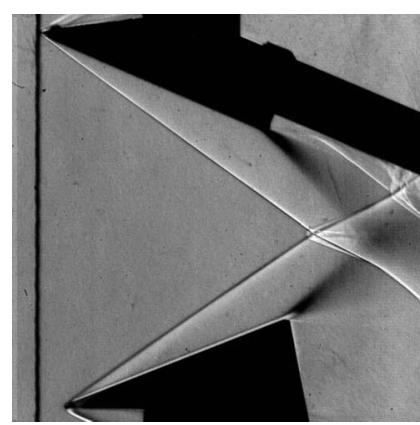

(d)
FIG. 21. Tunnel disturbances, such as dust, are capable of tripping the flow from regular reflection to the Mach reflection. Frame (b) shows a small piece of dust near the center line. After the piece of dust crosses the incident shocks transition to the Mach reflection begins. Images taken with a $3 \mu \mathrm{s}$ exposure and $121 \mu \mathrm{s}$ between frames. $M=4, \quad \alpha_{\text {lower }}=33.9^{\circ}$, and $\alpha_{\text {upper }}=36.4^{\circ}$.

of the speed of the object, it is only seen in one frame. Immediately after this object leaves the field of view transition to the Mach reflection begins.

\section{EXPERIMENTAL MACH STEM HEIGHTS}

Table II gives the Mach stem height results for various upper wedge angles from the current experiments. The results shown in Table II are plotted in Fig. 22, along with the theoretical calculations of Mouton and Hornung, ${ }^{11}$ the previous experimental results of Hornung and Robinson, ${ }^{12}$ and the current Amrita calculations. Very good agreement between the theoretical, computational, and current experimental

TABLE II. Mach stem heights measured at various upper wedge angles. $\alpha_{\text {equiv }}$ represents the symmetric shock angle that would produce an equivalent Mach stem height, based on the asymmetric theory of Mouton (Ref. 2).

\begin{tabular}{lllll}
\hline \hline$\alpha_{\text {upper }}$ & $\alpha_{\text {lower }}$ & $\alpha_{\text {equiv }}$ & $g / w$ & $s / w$ \\
\hline 34.7 & 33.6 & 34.1 & 0.395 & 0.027 \\
35.8 & 34.4 & 35.1 & 0.394 & 0.047 \\
36.8 & 33.5 & 35.1 & 0.391 & 0.055 \\
39.6 & 33.3 & 36.2 & 0.383 & 0.099 \\
39.9 & 33.7 & 36.5 & 0.384 & 0.106 \\
43.0 & 33.5 & 37.6 & 0.380 & 0.146 \\
42.8 & 34.2 & 37.9 & 0.381 & 0.147 \\
\hline \hline
\end{tabular}




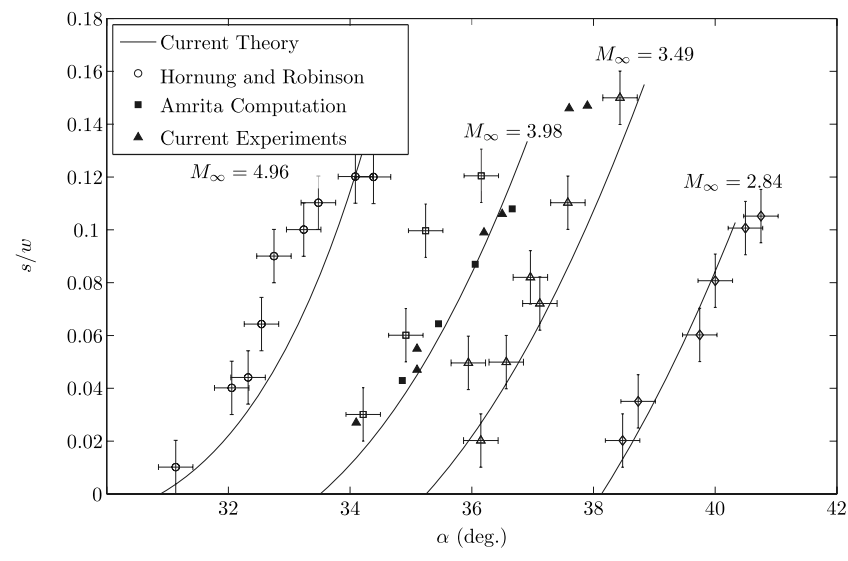

FIG. 22. Comparison of current experimental Mach stem height results against theoretical estimates by Mouton and Hornung (Ref. 11), measurements by Hornung and Robinson (Ref. 12), as well as current computational work done using Amrita. $\gamma=1.4$ and $g / w \approx 0.4$.

work is seen. The experimental work of Hornung and Robinson consistently showed higher Mach stem heights than the current experiments.

\section{EXPERIMENTAL MACH STEM GROWTH}

A theoretical growth rate for a Mach stem starting at regular reflection is presented by Mouton and Hornung. ${ }^{11}$ The growth rate can be measured from the new experimental data. With the wedges in the dual-solution domain and with initial regular reflection, energy was deposited on the lower wedge, as discussed in Sec. IV B. The deposition of energy causes the flow to trip from regular to Mach reflection. Since the initial flow is inside the dual-solution domain, where the steady-state Mach stem height is finite, the Mach stem quickly grows to this steady-state height.

Figure 23 shows the measured Mach stem heights as a function of time after energy deposition together with the theoretical estimate. The theoretical estimate is based on the two-dimensional model presented by Mouton and Hornung; ${ }^{11}$ however, the experimental results are three dimensional The experiments show a near linear growth rate of the Mach stem to the steady-state height. The initial rapid

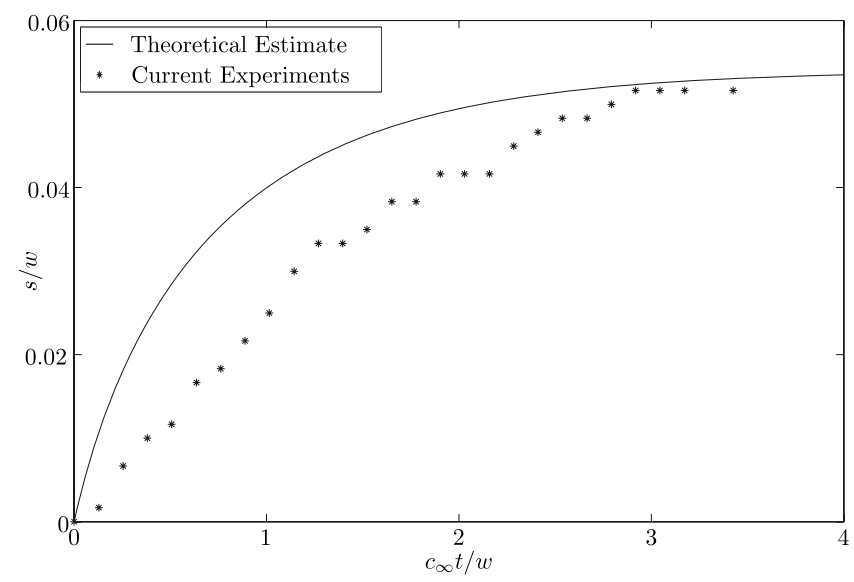

FIG. 23. Comparison of current experimental Mach stem growth rates with theoretical estimate. $\gamma=1.4$ and $g / w \approx 0.4$. growth of and subsequent nonlinear approach to the steadystate height predicated by the theory is not fully observed, although there is good agreement with experiment for the steady-state height and the time to reach it. The video from which the growth rate was measured was recorded with $38 \mu$ s between frames.

\section{CONCLUSIONS}

The Ludwieg tube facility at the California Institute of Technology was retrofitted with a Mach 4.0 nozzle. This Mach number is large enough to provide a sufficiently large dual-solution domain, while being small enough not to require preheating of the gas. The test time of the facility is $100 \mathrm{~ms}$, which requires the use of high-speed cinematography and a fast motor to rotate one of the two wedges.

The first experiments conducted on shock reflection in the Ludwieg tube verified the hysteresis phenomenon. The ability to enter the dual-solution domain with regular reflection is a qualitative measure of the quietness of the facility. The experiments show that in the Ludwieg tube facility, regular reflection could be maintained till approximately halfway between the von Neumann condition and the detachment condition.

Because of the complexities associated with particles impacting the wedge, and for experimental simplicity, it is possible to consider the impact of a particle on the wedge as a form of energy deposition. A theoretical estimate of the minimum energy required to cause transition from regular reflection to the Mach reflection is presented. This limit is calculated based on the condition that the blast wave from the energy deposition must be sufficiently strong to reach, and therefore influence, the transition point. An exact solution for strong shocks and an Euler computation for weak shocks are combined to calculate the minimum energy required for the energy deposition to influence the reflection point. This estimate of minimum required energy is compared with numerical results and very good agreement is seen when the energy is deposited close to the reflection point.

Energy deposition studies were performed using a 200 $\mathrm{mJ}$ pulsed Nd:YAG (yttrium aluminum garnet) laser focused on the surface of one of the wedges. The disturbance caused by the blast wave from the energy deposition is seen to affect the incident shock, and in some cases causes transition from regular to the Mach reflection. The location on the wedge where the energy is deposited is found to be an important criterion in whether or not transition occurs. This finding is consistent with the numerical work presented, which shows that the energy required to cause transition is dependent on the location where it is deposited. Future studies should measure the amount of energy deposited on the wedge so that an accurate minimum energy for transition can be calculated as a function of deposition location. A good way to measure the energy deposited is to visualize the blast wave caused by the energy deposition. Attempts to do this in the current experiments were unsuccessful because the densities required to 
visualize the blast wave were so high that the wave generated was too weak, i.e., the generated wave was a Mach wave.

Experiments were also performed to measure the Mach stem height and its growth rate. These results are compared with the theoretical estimates. Good agreement between the steady-state Mach stem height and the theoretical estimate is seen. Comparisons of Mach stem growth rate with theoretical estimates show significant differences, but do show good agreement in the time required to reach the steady-state height. The reasons for these differences are unknown, and may be attributable to three-dimensional effects in addition to the simplifying assumptions of the model.

\section{ACKNOWLEDGMENTS}

This work was funded by the Air Force Office of Scientific Research under the supervision of Dr. John Schmisseur. A portion of Christopher Mouton's studies were funded by the National Defense Science and Engineering Grant. Special thanks to Bahram Valiferdowsi for his extensive help in the design, manufacture, and repair of the Ludwieg tube facility. Thanks also to James Quirk for his help on the computational aspects of this work, and to Nicolas Ponchaut for his review of this work. The nozzle contour was designed by John Korte of NASA Langley Research Center.
${ }^{1}$ G. Ben-Dor, Shock Wave Reflection Phenomena (Springer-Verlag, New York, 2007).

${ }^{2} \mathrm{C}$. A. Mouton, "Transition between regular reflection and Mach reflection in the dual-solution domain," Ph.D. thesis, California Institute of Technology.

${ }^{3}$ H. G. Hornung, H. Oertel, and R. J. Sandeman, "Transition to Mach reflection of shock-waves in steady and psuedo-steady flow with and without relaxation," J. Fluid Mech. 90, 541 (1979).

${ }^{4}$ M. S. Ivanov, S. F. Gimelshein, and A. E. Beylich, "Hysteresis effect in stationary reflection of shock-waves," Phys. Fluids 7, 685 (1995).

${ }^{5}$ M. S. Ivanov, A. N. Kudryavtsev, S. B. Nikiforov, D. V. Khotyanovsky, and A. A. Pavlov, "Experiments on shock wave reflection transition and hysteresis in low-noise wind tunnel," Phys. Fluids 15, 1807 (2003).

${ }^{6}$ N. Sudani, M. Sato, T. Karasawa, J. Noda, A. Tate, and M. Watanabe, "Irregular effects on the transition from regular to Mach reflection of shock wave in wind tunnel flows," J. Fluid Mech. 459, 167 (2002).

${ }^{7}$ M. Y. El-Naggar, J. T. Klamo, M.-H. Tan, and H. G. Hornung, "Experimental verification of the Mach-number field in a supersonic Ludwieg tube," AIAA J. 42, 1721 (2004).

${ }^{8}$ J. J. Quirk, "AMRITA: A computational facility (for CFD modelling)," in 29th Computational Fluid Dynamics, von Karman Institute Lecture Series, Rhode-Saint-Genèse, Belgium, 1998, edited by H. Deconinck, Vol. 3, pp. D1-D72 (available online at http://www.amrita-cfd.org/cgi-bin/doc/vki).

${ }^{9}$ D. V. Khotyanovsky, A. Kudryavtsev, and M. S. Ivanov, "Effects of a single-pulse energy deposition on steady shock wave reflection," Shock Waves 15, 353 (2006).

${ }^{10}$ L. I. Sedov, Similarity and Dimensional Methods in Mechanics, 4th ed. (Academic, New York, 1959).

${ }^{11}$ C. A. Mouton and H. G. Hornung, "Mach stem height and growth rate predictions," AIAA J. 45, 1977 (2007).

${ }^{12}$ H. G. Hornung and M. L. Robinson, "Transition from regular to Mach reflection of shock-waves part 2. The steady-flow criterion," J. Fluid Mech. 123, 155 (1982). 